University of Nebraska - Lincoln

DigitalCommons@University of Nebraska - Lincoln

The coupling of a reflectron time-of-flight mass spectrometer with a cosmic simulation chamber: A powerful new tool for laboratory astrophysics

\author{
Claire L. Ricketts \\ NASA-Ames Research Center, Claire.L.Ricketts@nasa.gov \\ Cesar S. Contreras \\ NASA-Ames Research Center, Cesar.Contreras@nasa.gov \\ Robert L. Walker \\ NASA-Ames Research Center, Robert.L.Walker@nasa.gov \\ Farid Salama \\ NASA-Ames Research Center, farid.salama@nasa.gov
}

Follow this and additional works at: https://digitalcommons.unl.edu/nasapub

Part of the Physical Sciences and Mathematics Commons

Ricketts, Claire L.; Contreras, Cesar S.; Walker, Robert L.; and Salama, Farid, "The coupling of a reflectron time-of-flight mass spectrometer with a cosmic simulation chamber: A powerful new tool for laboratory astrophysics" (2011). NASA Publications. 84.

https://digitalcommons.unl.edu/nasapub/84

This Article is brought to you for free and open access by the National Aeronautics and Space Administration at DigitalCommons@University of Nebraska - Lincoln. It has been accepted for inclusion in NASA Publications by an authorized administrator of DigitalCommons@University of Nebraska - Lincoln. 


\title{
The coupling of a reflectron time-of-flight mass spectrometer with a cosmic simulation chamber: A powerful new tool for laboratory astrophysics
}

\author{
Claire L. Ricketts, Cesar S. Contreras, Robert L. Walker, Farid Salama* \\ NASA-Ames Research Center, Mail Stop 245-6, Space Science \& Astrobiology Division, Astrophysics Branch, Moffett Field, CA 94035, USA
}

\section{A R T I C L E I N F O}

\section{Article history:}

Received 9 October 2010

Received in revised form

23 November 2010

Accepted 24 November 2010

Available online 3 December 2010

\section{Keywords:}

Reflectron time-of-flight mass

spectrometry (ReTOF-MS)

Pulsed discharge nozzle (PDN) source

Cavity ringdown spectroscopy (CRDS)

Polycyclic aromatic hydrocarbon (PAH)

Planetary atmospheres

Interstellar medium (ISM)

\begin{abstract}
A B S T R A C T
The addition of an orthogonal reflectron time-of-flight mass spectrometer (ReTOF-MS) to the NASA Ames cosmic simulation chamber (CSC) experiment is described. The simulation chamber contains the elements that produce the molecular species under astrophysically relevant conditions. A pulsed discharge nozzle (PDN) produces ions, neutrals and radicals in a plasma discharge, which are then expanded and supersonically cooled into the chamber. The coupling of the ReTOF-MS to the CSC provides real-time identification of the species, including cations and neutrals, formed in the plasma, an insight into the chemical pathways of the species reacting in the plasma, and an efficient method for the quick determination of the species present in the plasma, which can then be probed spectroscopically with the cavity ring-down spectrometer (CRDS). The combination of the ReTOF-MS, CRDS and PDN components into a single instrument offers a powerful tool, which can be used to probe a variety of different astrophysical environments such as interstellar clouds and planetary atmospheres. The experimental details and representative mass spectra are presented for plasmas generated in combinations of argon and methane samples. These mass spectra show the unambiguous detection of externally generated ions from the plasma in the simulation chamber. In addition, the various spectra show evidence of fragmentation and bond forming reactivity, illustrating the impact of the composition of the plasma.
\end{abstract}

Published by Elsevier B.V.

\section{Introduction}

Carbon species play an important role in the Universe, in environments as diverse as dense and diffuse interstellar medium (ISM) clouds, circumstellar disks and planetary atmospheres [1]. Polycyclic aromatic hydrocarbons (PAHs) are considered to be an important component of extraterrestrial carbon materials, so extensive experimental, theoretical and observational research has been dedicated to the study of PAHs for over twenty-five years [2-4]. One such experiment is the pulsed discharge nozzle (PDN)-cavity ring-down spectrometer (CRDS) system, a cosmic simulation experiment, which was developed at NASA-Ames to measure the gas phase spectra of neutral and ionized interstellar PAH analogs [5]. The PDN-CRDS has been successfully used to measure the spectra of several PAH species, for example the naphthalene cation $\left(\mathrm{C}_{10} \mathrm{H}_{8}{ }^{+}\right)$and acenaphthene cation $\left(\mathrm{C}_{12} \mathrm{H}_{10}{ }^{+}\right)$ [5], pyrene cation $\left(\mathrm{C}_{16} \mathrm{H}_{10}{ }^{+}\right)$[6], neutral perylene $\left(\mathrm{C}_{20} \mathrm{H}_{12}\right)$ [7], and benzo $(\mathrm{g}, \mathrm{h}, \mathrm{i})$ perylene $\left(\mathrm{C}_{22} \mathrm{H}_{12}\right)$ [8], under the conditions of the interstellar medium.

\footnotetext{
* Corresponding author. Tel.: +1 650604 3384; fax: +1 6506046779.

E-mail addresses: Claire.L.Ricketts@nasa.gov (C.L. Ricketts), Cesar.Contreras@nasa.gov(C.S. Contreras),Robert.L.Walker@nasa.gov(R.L.Walker), Farid.Salama@nasa.gov (F. Salama).
}

The cosmic simulation chamber (CSC) plasma experiments were originally dedicated to the formation and the characterization of free, cold, neutral and ionized interstellar PAH analogs using cavity ringdown spectroscopy to obtain optical spectra measurements. However, the observation of the formation of soot in the chamber and on the electrodes of the discharge nozzle, as well as observed fragmentation of the precursor PAH molecules when the applied discharge voltage was increased above a certain threshold, implied the need for a real-time monitoring of the plasma products [9]. The addition of an orthogonal reflectron time-of-flight mass spectrometer (ReTOF-MS) to the simulation chamber (CSC) allows the full identification of the mass and charge of all molecular species, both ionic and neutral, entering the ReTOF-MS from the external ion source [10-14]. The ReTOF-MS will also act as a complementary technique for the CRDS by providing an important insight in realtime into the species present in the discharge, which can then be individually selected and optimized for further analysis with the CRDS. This combination of the PDN-CRDS system with a ReTOFMS is a powerful technique that provides a unique opportunity to probe molecules and ions in cold astrophysically relevant plasma and expansion conditions in the laboratory.

The PDN-CRDS-ReTOF-MS combination has been developed as a tool to shed light on astrophysical environments, specifically those involving carbon species, by addressing many unanswered questions such as those regarding planetary atmospheres, inter- 
stellar clouds and the outflows of carbon stars. Typical examples of such open questions are the unexplained growth of large species in the $\mathrm{N}_{2} / \mathrm{CH}_{4}$ atmosphere of Titan recently detected by Cassini [15], the important role that positive and negative organic ions play in the chemical network of interstellar clouds [16], and the formation mechanisms of circumstellar and interstellar carbon grains [17]. The PDN-CRDS-ReTOF-MS experiment is a unique combination of techniques; this paper will discuss the coupling of the ReTOF-MS to the existing PDN-CRDS experiment, with representative results. The instrument is first described in detail followed by a discussion of the mass spectra measured of the ions formed in the PDN, otherwise referred to as discharge slit nozzle. In conclusion, an assessment is provided of this new instrument that combines gaseous ions formed in a cold plasma (PDN element), optical spectroscopy (CRDS element) and mass spectrometry (ReTOF-MS element).

\section{Instrumentation}

In this section we describe the simulation chamber (CSC), which houses the pulsed discharge slit nozzle (PDN) and cavity ring-down spectrometer (CRDS) components of the experimental system, and the orthogonal ReTOF-MS. A schematic of the experimental arrangement of the combined components is shown in Fig. 1. The entire PDN-CRDS-ReTOF-MS apparatus consists of three vacuum chambers; the simulation chamber (CSC), which houses the slit nozzle (PDN) and the ring-down cavity mirrors, the transition chamber and the ReTOF-MS chamber. The transition chamber and ReTOF-MS chamber form one complete assembly and are mounted directly opposite the exit of the simulation chamber. One of the unique features of this experimental set-up is that the transition and ReTOF-MS chambers are attached to the simulation chamber via a reinforced stainless steel rotating door (MDC Vacuum Products) that was custom-designed to support the combined weight of the transition chamber and ReTOF-MS assembly. Therefore, the assembly can be swung round, away from the simulation chamber, in order to allow face on access to the slit nozzle, for example for maintenance or to place a window in the port for the viewing of the plasma generated by the PDN.

\subsection{The cosmic simulation chamber (CSC)}

The PDN and the CRDS components of the experimental system were developed in collaboration with Los Gatos Research Inc. and have been discussed in detail elsewhere [5,7], so only a brief description will be given here.

\subsubsection{Pulsed discharge nozzle (PDN) ion source}

The PDN source produces a plasma discharge, which is expanded to astrophysically relevant pressure/temperature regimes upon which the products of the expansion can be probed by CRDS or ReTOF-MS or a combination of both techniques. The dynamics of the free jet expansion and the nature of the plasma generated by the slit nozzle are well characterized [18-20]. The PDN source is part of the original experimental configuration and is vacuum pumped using an Edwards Blower pump (Edwards, EH-1200) that is backed by a dry pump (Edwards, GV-250) to approximately $10^{-3}$ Torr. The pumping capacity of the resulting system is $250 \mathrm{l} / \mathrm{s}$. The pulsed discharge nozzle consists of a heated copper sample reservoir and a $10 \mathrm{~cm}$-long by $200 \mu \mathrm{m}$-wide slit, which is sealed from inside by a Vespel (Dupont, SP-22 Grade) slit poppet driven by three synchronized pulsed solenoid valves (General Valve Series 9). Two stainless knife-edge electrodes are mounted outside the PDN on each side of the slit. The two electrodes are separated by an even gap of $400 \mu \mathrm{m}$ and configured as the cathode of a high-voltage pulse generator. The pulsed discharge nozzle (PDN) assembly itself is configured as the anode. This design enables the generation of atomic and molecular ions and radicals in the jet expansion by application of a high voltage typically between $-500 \mathrm{~V}$ and $-1000 \mathrm{~V}$ as the carrier gas is introduced into the slit nozzle. For samples solid at room temperature (e.g., PAHs), the sample is placed on the bottom of the sample reservoir and the entire block is heated to ensure adequate evaporation. Heating of the reservoir has been shown not to alter the expansion temperature of the gas [5]. Previous studies gave a rotational temperature of $52 \mathrm{~K}$ for the gas in the supersonic expansion, measured $4 \mathrm{~mm}$ from the opening of the slit [7]. The positioning of the slit of the PDN can be adjusted horizontally and vertically from the outside of the chamber and has been adjusted to align with the opening orifice, a skimmer, of the ReTOF-MS instrument (Fig. 1). A recent modification to the PDN assembly allows the entire PDN to be moved to varying distances from the opening orifice to the ReTOF-MS instrument, in order to probe different regions of the expansion.

\subsubsection{Cavity ringdown spectroscopy (CRDS)}

The supersonic beam expansion containing the carrier gas seeded with the sample is probed by cavity ringdown spectroscopy (CRDS). To briefly summarise, in the CRDS experiments the spectra of ionic and neutral species are probed using a Nd:yttrium aluminum garnet (YAG) (Quanta-Ray Lab 150 from Spectra-Physics) pumped dye laser (Quanta-Ray PDL-2 from Spectra-Physics). The ring-down cavity consists of two high-reflectivity (99.999\%) concave ( $6 \mathrm{~m}$ curvature radius) mirrors (Los Gatos Research) mounted $55 \mathrm{~cm}$ apart. The ring-down signal is collected by a photosensor module (H6780-04 from Hamamatsu) and digitized by a $20-\mathrm{MHz}$ 12-bit acquisition board (Adlink, PCI9812). A personal computer program then processes the data and the cavity losses are extracted.

\subsection{Reflectron time-of-flight mass spectrometer (ReTOF-MS)}

The components of the ReTOF-MS chamber have been custom built by Jordan TOF products, and assembled, modified and commissioned at NASA-Ames. The design of the instrument is based on a modified version of the original concept of the spectrometer [21]. The chamber houses the ReTOF-MS assembly, which includes the ion extraction optics region, a supplementary electron ionization source, ion focusing and deflecting electrostatic lenses, the field free drift tube, the reflecting optics assembly and a $40 \mathrm{~mm}$ diameter multi channel plate (MCP) detector. There is an optional $18 \mathrm{~mm}$ diameter MCP detector mounted behind the reflectron for use in the linear mode. The MCP signal is amplified using an ORTEC preamplifier (VT-120) and mass spectra are recorded using an ORTEC 'FastFlight-2' digital signal analyzer, with a PC driven acquisition program. In conjunction with a DG-535 signal generator (Stanford Instruments), the 'FastFlight-2' also provides the synchronization of the detection with the extraction pulse voltage that introduces the ions into the ReTOF-MS drift tube.

\section{Experimental}

In this section we describe the coupling of the ReTOF-MS to the cosmic simulation chamber (CSC), with details of the transition chamber and the detection of ions and neutrals formed in the simulation chamber.

\subsection{Coupling the reflectron time-of-flight mass spectrometer (ReTOF-MS) to the cosmic simulation chamber (CSC)}

A custom-built ReTOF-MS (Jordan TOF, Inc.) has been coupled with the simulation chamber, in order to separate and detect all products produced in the slit discharge nozzle (PDN). The following 


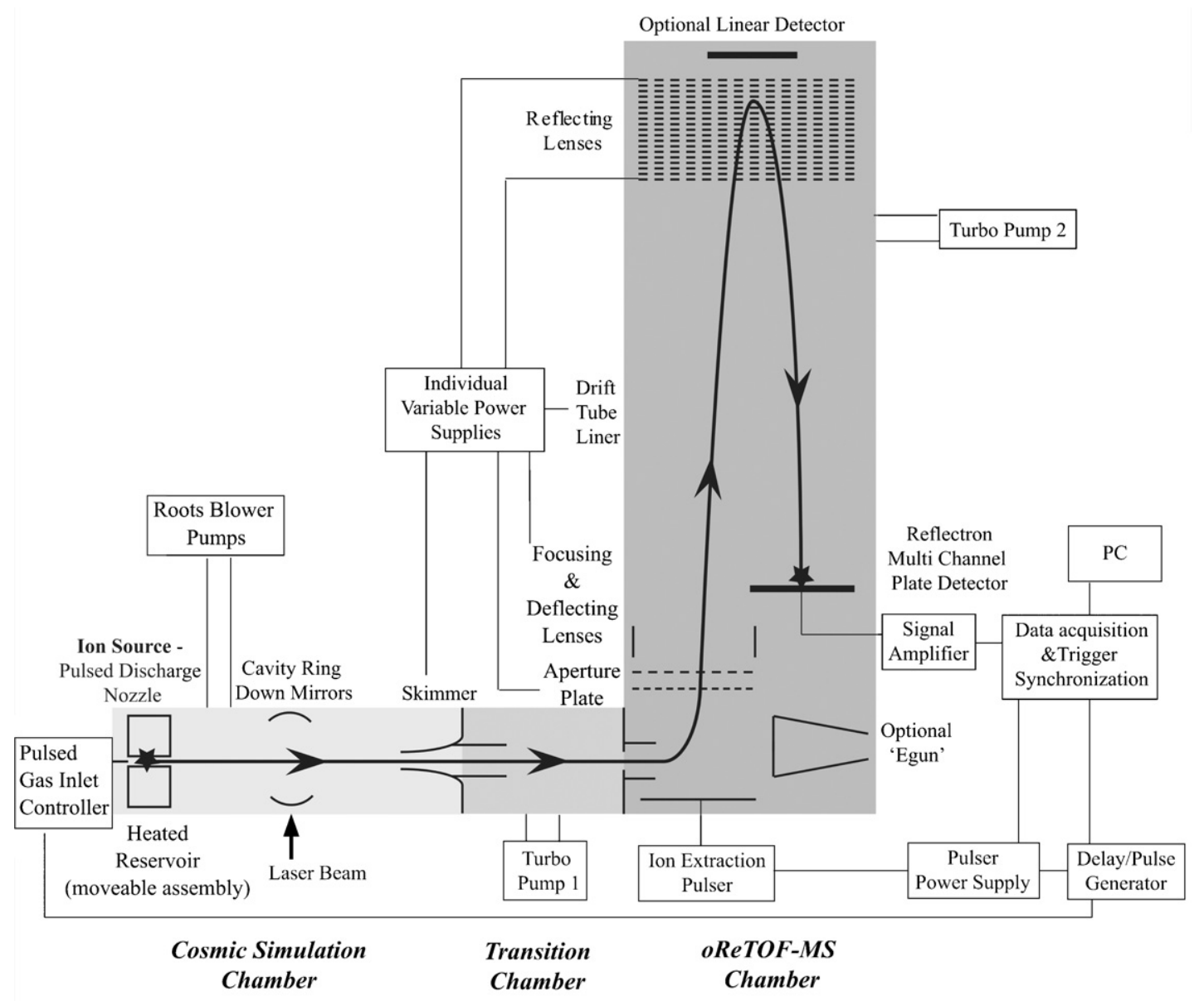

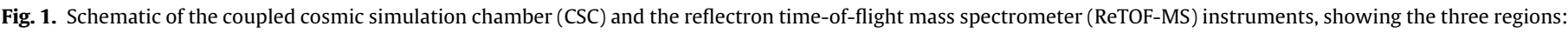

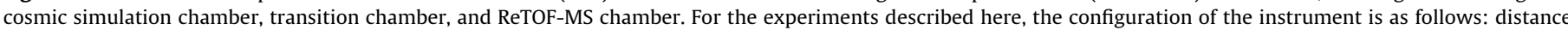

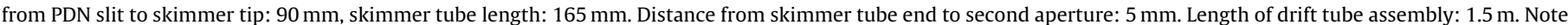
that the components are not drawn to scale.

sections serve to describe the coupling of the CSC to the ReTOFMS (Fig. 1). To briefly overview, the ionic and neutral products generated in the plasma of the slit nozzle discharge (PDN), which may also consist of the unreacted precursor species, are expanded into the simulation chamber. A fraction of these species will travel through the aperture of a skimmer opposite the output of the PDN. The species then continue through a second aperture and into the extraction region of the ReTOF-MS. From the extraction region the ions are accelerated, perpendicular to their original direction of motion, and focused into the drift tube of the ReTOF-MS towards the reflecting assembly of the ReTOF-MS, where the reflecting lenses turn the ions by approximately $180^{\circ}$ and the ions continue to drift to the multi channel plate (MCP) detector.

\subsubsection{Transition chamber}

The pressure regime of the simulation chamber is of the order of $10^{-3}$ Torr while the ReTOF-MS requires a low pressure environment $\left(10^{-6}\right.$ Torr) to minimize any disturbance in the ion's flight path. To reach the required pressure regime in the ReTOF-MS, differential pumping is required and the simulation chamber that contains the PDN ion source is linked to a transition chamber via a skimmer. The transition chamber $\left(10^{-4}\right.$ Torr $)$ is in turn connected to the ReTOF-MS chamber via a second aperture. This pressure gradient was achieved by accurately determining the optimal size of the apertures on the skimmer and the plate ( 2 and $3 \mathrm{~mm}$ aperture, respectively), allowing for good pressure control in the chambers and high ion signal. The base pressure of the transition cham- ber is maintained below $2 \times 10^{-8}$ Torr when no gas is injected and below $9 \times 10^{-4}$ Torr when the discharge slit nozzle (PDN) is in operation. For comparison, the base pressure of the ReTOF-MS chamber is below $2 \times 10^{-8}$ Torr with no injected gas and typically $2 \times 10^{-6}$ Torr when the PDN is operating. Vacuum in the transition chamber is achieved using a turbomolecular pump (Varian TV301 NAV) with gate valves and controllers, backed by a dry rotary pump (Franklin Electric 1201006416). The vacuum in the ReTOFMS chamber is maintained using a second turbomolecular pump (Varian TV551 NAV), backed by a dry rotary pump (Franklin Electric 1201006416) and the pressure is monitored using a cold cathode gauge.

Both the skimmer and the plate are connected to external power supplies in order to polarize and hence actively attract the ions generated in the plasma. Typically the skimmer provides the optimum transport of the ions to the ReTOF-MS when the voltage applied to the skimmer is between $0 \mathrm{~V}$ and $-2 \mathrm{~V}$. The nickel-plated skimmer is mounted on a stainless steel tube. The length of the tubing and the distance the tubing and skimmer penetrates into either the transition chamber or simulation chamber can be varied. For these experiments the PDN slit to skimmer tip distance was $90 \mathrm{~mm}$, with the skimmer-tube total length being $165 \mathrm{~mm}$ and the skimmer tube end to second aperture gap was $5 \mathrm{~mm}$. The flat plate, with an aperture, at the entrance to the ReTOF-MS chamber is mounted on a tube, which is isolated from the other electrostatic optics in order to apply a negative voltage between $-20 \mathrm{~V}$ and $-30 \mathrm{~V}$ to attract positive ions from the plasma. 


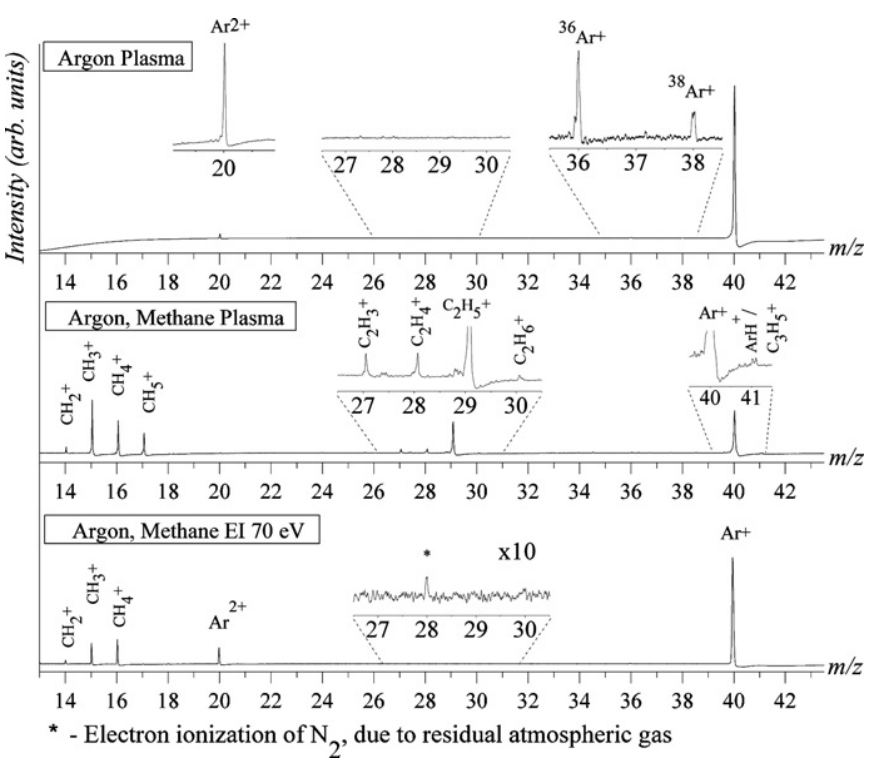

Fig. 2. The two top spectra are for mass spectra of ions externally generated in the pulsed slit nozzle discharge (PDN) from argon plasma $(-800 \mathrm{~V})$ for the top spectrum, and argon/methane plasma $(-800 \mathrm{~V})$ for the middle spectrum. The bottom spectrum is the electron ionization (EI) mass spectrum of ions generated internally from the neutral precursor gas mixture. Note: in the plasma experiments, the ions are externally generated before extraction in the ReTOF-MS and the Egun is not used.

\subsubsection{Detection of ions formed in the CSC with the ReTOF-MS}

The pulsed discharge nozzle (PDN) acts as the ionization source in this experiment, and the main purpose of the ReTOF-MS is to detect these externally generated ions. After the ions from the simulation chamber fly through the skimmer into the transition chamber, they travel through an aperture with an applied attractive electrostatic potential ( $-30 \mathrm{~V}$ for positive ion detection) to reach the extraction region of the ReTOF-MS. To reiterate, transporting ions from the PDN requires differential pumping in the transition and ReTOF-MS chambers, as well as the application of an appropriate voltage to the transporting skimmer, aperture plate and the extraction plate in the extraction region of the ReTOF-MS. In the extraction region of the ReTOF-MS, a bias voltage of $-30 \mathrm{~V}$ is set to allow ions to enter the extraction region. Timing of these events is also synchronized. After the gas pulse occurs to introduce the carrier gas into the pulsed discharge nozzle (PDN), the high voltage is applied on the PDN electrodes to create the plasma-generated ions. The discharge event lasts $\sim 300 \mu \mathrm{s}$, and occurs in the middle of the gas pulse event. Ions formed in the plasma are thus provided ample time to travel to the extraction region before sampling in the ReTOF-MS. From the extraction region of the ReTOF-MS the ions are orthogonally injected, typically by a pulsed voltage of $+200 \mathrm{~V}$ applied to the repeller plate, and focused into the drift tube of the ReTOF-MS. At the end of the drift tube the ions are back-reflected, and hence focused, before detection using the MCP detector. The FastFlight-2 hardware monitors all events after the extraction pulse, with the MCP detection time typically set to collect mass spectra up to $2000 \mathrm{~m} / \mathrm{z}$. Although all major peaks are identifiable from single-shot spectral scans, averages of 3000 scans per spectrum are routinely taken to optimize signal-to-noise. The high sensitivity of the instrument is illustrated in Fig. 2, which shows the detection of the peaks associated with the main isotopes of argon. The ${ }^{38} \mathrm{Ar}^{+}$and ${ }^{36} \mathrm{Ar}^{+}$isotope peaks, present at a natural mole fraction of $6 \times 10^{-4}$ and $3 \times 10^{-3}$, respectively, are clearly recognized.

\subsubsection{Detection of neutrals formed in the CSC with the ReTOF-MS}

Neutral species, which can be relevant to astrophysical applications, are also generated in the plasma and travel with the ions in the cold expansion. Neutral PAHs, for example, have been detected and probed with CRDS $[7,8]$. Hence, the ReTOF-MS is equipped with an electron ionization (EI) source that ionizes the cold neutrals in the extraction region of the ReTOF-MS. The EI source uses a tungsten filament to produce electrons at $70 \mathrm{eV}$ (tunable from 40 to $80 \mathrm{eV}$ ), which in turn produce ions via electron-neutral collisions. When operating in the EI or internally generated ion mode, the neutrals travel from the simulation chamber into the ReTOFMS without the application of voltage to the skimmer or plate in the transition chamber, with the bias of the ReTOF-MS extraction region set to ground. Since the extraction plate is set to ground, only ions formed internally with EI are detected. The timing and pulsing procedures with the repeller plate and the introduction of the internally generated ions into the drift tube is similar to that described for the externally generated ions.

\section{Results and discussion}

To demonstrate the ability to use the ReTOF-MS to detect ions that are externally generated, a variety of plasmas have been probed. These include discharge expansions from single gases for example argon, nitrogen and methane, from a mixture of these gases and also from these gases seeded with selected PAHs. In this article we present mass spectra of ions detected from two different plasma compositions, an argon-only and an argon/methane mixture, as representative mass spectra of the measurements that can be obtained with this new instrument. The mass spectra recorded from the argon-only plasma and argon/methane plasma are shown in Fig. 2 and are discussed in the next section. Future reports will focus on the astrophysical applications of the measurements.

\subsection{Real-time detection of ions generated in the CSC with the ReTOF-MS}

In the current experimental arrangement, the mass resolution, for the spectra of externally generated ions, is approximately 650 (FWHM) based on $R=m / \Delta m$. To briefly summarize the spectra in Fig. 2 , in the argon plasma $(-800 \mathrm{~V})$ we identify the peaks in the mass spectrum as $\mathrm{Ar}^{2+}$ and $\mathrm{Ar}^{+}$, including isotopes ${ }^{36} \mathrm{Ar}^{+}$and ${ }^{38} \mathrm{Ar}^{+}$, demonstrating the ability to detect externally generated ions, even those likely to account for a small percentage of the total ionization cross-section such as the argon isotopes. In the argon/methane plasma $(-800 \mathrm{~V})$ we identify the peaks $\mathrm{Ar}^{+}$and $\mathrm{CH}_{4}{ }^{+}$from the ionization of parent compounds. Also detected are peaks associated with hydrogen loss, $\mathrm{CH}_{2}{ }^{+}, \mathrm{CH}_{3}{ }^{+}$, and hydrogen gain, $\mathrm{CH}_{5}{ }^{+}$, from the parent $\mathrm{CH}_{4}$. Additionally, we observe bond formation reactivity, or 'growth' of the carbon species, in the argon/methane plasma, with peaks assigned to $\mathrm{C}_{2} \mathrm{H}_{3}{ }^{+}, \mathrm{C}_{2} \mathrm{H}_{4}{ }^{+}, \mathrm{C}_{2} \mathrm{H}_{5}{ }^{+}, \mathrm{C}_{2} \mathrm{H}_{6}{ }^{+}$, and potentially $\mathrm{ArH}^{+}$or $\mathrm{C}_{3} \mathrm{H}_{5}{ }^{+}$. The clear evidence of bond formation from the argon/methane plasma demonstrates that ionic products formed in reaction processes within the plasma are stable or are stabilized by the cold expansion and survive in order to be detected in the ReTOF-MS, a significant distance from the ionization region.

An electron ionization (EI) mass spectrum of the same neutral argon/methane gas mixture precursor was taken for comparison, and is shown in Fig. 2. A clear distinction can be seen when comparing the two cases. The EI experiment produces the parent molecular ion and some fragment ions, while the plasma experiments also produce ions due to recombination.

\section{Conclusion}

We have coupled a cosmic simulation chamber with a pulsed plasma discharge to an orthogonal reflectron time-of-flight mass spectrometer. Using the ReTOF-MS we can directly detect, in 
real-time, the ions generated in the simulation chamber under astrophysical conditions. Now with the mass spectral information obtained, other species besides the molecular ion formed in the plasma can be probed spectroscopically with cavity ringdown spectroscopy (CRDS). This capability was necessary in order to proceed with the CRDS experiments. Mass spectra recorded from an argononly plasma and an argon/methane plasma demonstrate the power of this coupling, showing unambiguous detection of externally generated ions as well as evidence of bond-forming reactivity within the plasma.

\section{Acknowledgments}

This work is supported by NASA. We acknowledge the support provided by the NASA Science Mission Directorate Planetary Atmospheres, Cosmochemistry and Astronomy and Physics Research and Analysis (APRA) Programs. C.L.R. and C.S.C. acknowledge the support of the NASA Postdoctoral Program (NPP). The authors acknowledge fruitful discussions with Paul Jordan, Ludovic Biennier, Hassan Sabbah, Joseph Roser, Jerome Remy, Oscar Martinez, Veronica Bierbaum and Nigel Adams.

\section{References}

[1] Th. Henning, F. Salama, Carbon in the universe, Science 282 (1998) 2204-2210.

[2] F. Salama, in: S. Kwok, S.A. Sandford (Eds.), PAHs in Astronomy - A Review, Organic Matter in Space, IAU Symposium 251, Cambridge University Press, Cambridge, UK, 2008, pp. 357-365.

[3] A.G.G.M. Tielens, Interstellar polycyclic aromatic hydrocarbon molecules, Annual Review of Astronomy and Astrophysics 46 (1) (2008) 289-337.

[4] C.W. Bauschlicher, C. Boersma, A. Ricca, A.L. Mattioda, J. Cami, E. Peeters, F. Sánchez de Armas, G. Puerta Saborido, D.M. Hudgins, L.J. Allamandola, The NASA Ames polycyclic aromatic hydrocarbon infrared spectroscopic database: the computed spectra, The Astrophysical Journal: Supplement Series 189 (2010) 341-351.

[5] L. Biennier, F. Salama, L.J. Allamandola, J.J. Scherer, Pulsed discharge nozzle cavity ring down spectroscopy of cold PAH ions, The Journal of Chemical Physics 118 (2003) 7863-7872.

[6] L. Biennier, F. Salama, M. Gupta, A. O’Keefe, Multiplex integrated cavity output spectroscopy of cold pah cations, Chemical Physics Letters 387 (2004) 287-294.
[7] X. Tan, F. Salama, Cavity ring-down spectroscopy and theoretical calculations of the $\mathrm{S}_{1}\left(1 \mathrm{~B}_{3 \mathrm{u}}\right) \leftarrow \mathrm{S}_{0}\left(1 \mathrm{~A}_{\mathrm{g}}\right)$ transition of jet cooled perylene, The Journal of Chemical Physics 122 (2005), 084318 (1-8).

[8] X. Tan, F. Salama, Cavity ring-down spectroscopy and vibronic activity of jetcooled benzo[ghi]perylene, The Journal of Chemical Physics 123 (2005), 014312 $(1-7)$.

[9] L. Biennier, M. Hammond, J. Elsila, R. Zare, F. Salama, From organic molecules to carbon particles: implications for the formation of interstellar dust, astrochemistry: recent successes and current challenges, in: A.J Markwick-Kemper (Ed.), IAU Symposium, vol. 231, 2005.

[10] M. Guilhaus, D. Selby, V. Mlynski, Orthogonal acceleration time-of-flight mass spectrometry, Mass Spectrometry Reviews 19 (2000) 65-107.

[11] A.M. Ricks, G.E. Douberly, M.A. Duncan, The infrared spectrum of protonated naphthalene and its relevance for the unidentified infrared bands, The Astrophysical Journal 702 (2009) 301-306.

[12] S. Chakraborty, R. Omidyan, I. Alata, I.B. Nielsen, C. Dedonder, M. Broquier, C. Jouvet, Protonated benzene dimer: an experimental and ab initio study, Journal of the American Chemical Society 131 (2009) 11091-11097.

[13] H. Tanimoto, N. Aoki, S. Inomata, J. Hirokawa, Y. Sadanaga, Development of a PTR-TOFMS instrument for real-time measurements of volatile organic compounds in air, International Journal of Mass Spectrometry 263 (2007) $1-11$.

[14] E. Witkowicz, H. Linnartz, C. de Lange, W. Ubachs, A. Sfounis, M. Massaouti, M Velegrakis, Mass spectrometric and laser spectroscopic characterization of a supersonic planar plasma expansion, International Journal of Mass Spectrometry 232 (2004) 25-29.

[15] J.H. Waite Jr., D.T. Young, T.E. Cravens, A.J. Coates, F.J. Crary, B. Magee, J. Westlake, The process of tholin formation in Titan's upper atmosphere, Science 316 (2007) 870-875

[16] T.P. Snow, V.M. Bierbaum, Ion chemistry in the interstellar medium, Annual Review of Analytical Chemistry 1 (2008) 229-259.

[17] A.P. Jones, Interstellar and circumstellar grain formation and survival, philosophical transactions, Mathematical Physical and Engineering Sciences 359 (1787) (2001) 1961-1972.

[18] J. Remy, L. Biennier, F. Salama, Plasma in a pulsed discharge environment, fourth triennial special issue on images in plasma science, IEEE Transactions on Plasma Science 33 (2005) 554-555.

[19] B.H.P. Broks, W.J. Brok, J. Remy, J.J.A.M. van der Mullen, A. Benidar, L. Biennier, F. Salama, Numerical investigation of the discharge characteristics of the pulsed discharge nozzle, Physical Review E: Statistical Physics, Plasmas, Fluids and Related Interdisciplinary Topics 71 (2005), 036409 (1-9).

[20] L. Biennier, A. Benidar, F. Salama, Flow dynamics of a pulsed planar expansion, Chemical Physics 326 (2006) 445-457.

[21] D.M. Lubman, R.M. Jordan, Design for improved resolution in a time-of-flight mass spectrometer using a supersonic beam and laser ionization, Review of Scientific Instruments 56 (1985) 373-376. 